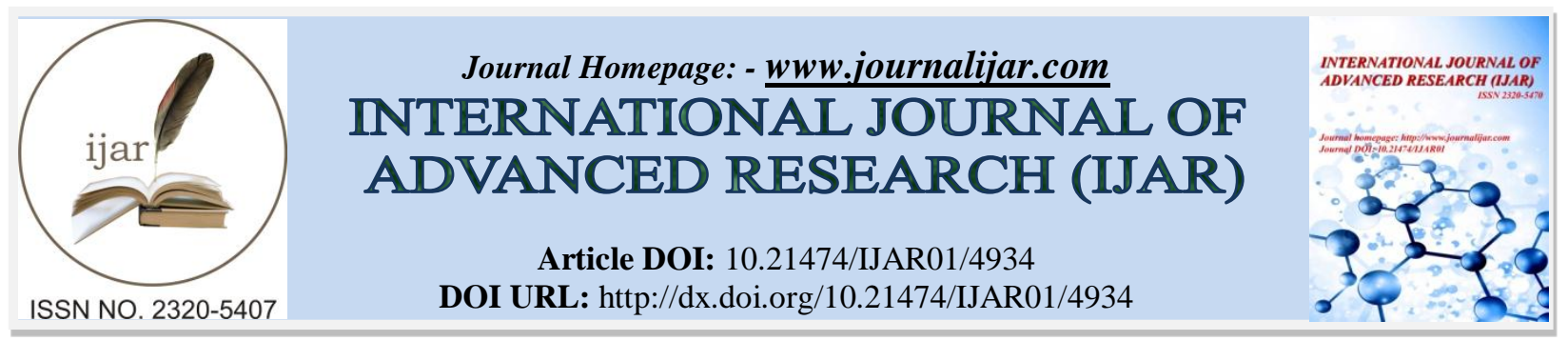

RESEARCH ARTICLE

\title{
OS NAVICULAIRE ACCESSOIRE SYMPTOMATIQUE UNILATERAL CHEZ L'ENFANT UNILATERAL SYMPTOMATIC ACCESSORY NAVICULAR BONE IN CHILDREN.
}

Mohamed Rachid Bensalah and Reda Ouazzaâ.

Service de Traumatologie-Orthopédie I, Hôpital Militaire d'Instruction Mohammed V, Université Mohammed V, Rabat, Maroc.

\section{Manuscript Info}

\section{Manuscript History}

Received: 23 May 2017

Final Accepted: 25 June 2017

Published: July 2017

Nous rapportons l'observation d'un enfant de 12 ans, sans antécédents, ayant présenté une douleur chronique progressive depuis six mois sur l'arche interne du pied gauche. S'intensifiant à la marche, et associée à une difficulté de chaussage.

A l'examen et au regard de l'os naviculaire, on note la présence d'un œdème et d'une inflammation, ainsi qu'une douleur à la palpation de la face médiale du pied.

La radiologie standard a montré la présence d'un os naviculaire accessoire gauche:-

Le scanner des deux pieds a objectivé un os naviculaire accessoire à gauche de type II, faisant une synchondrose avec l'os naviculaire, ainsi qu'une insertion des fibres du tendon tibial postérieur sur l'os accessoire, et l'absence de l'os accessoire à droite.

Une scintigraphie osseuse a montrée une hyperfixation intense au niveau du dôme et du col de l'astragale gauche.

Le traitement médical usuel a été prescrit, associé au port d'une orthèse plantaire et de chaussures adaptées. Une infiltration de corticoïdes a également été réalisée. Devant l'échec du traitement conservateur, un traitement chirurgical consistant en une arthrodèse de l'os naviculaire avec son accessoire à l'aide de vissage a été réalisé.

\section{L'appui immédiat et la rééducation précoce ont été indiqués:-}

L'os naviculaire accessoire est un os surnuméraire rare du tarse. Il est attaché par une synchondrose avec le corps principal de l'os naviculaire. Plus fréquent chez la femme jeune, souvent bilatérale et asymptomatique.

Le traitement est chirurgical, indiqué en cas d'échec du traitement médical, consiste soit en un forage percutané de la synchondrose, une arthrodèse de l'os naviculaire avec son accessoire, ou une simple excision de l'os naviculaire accessoire.

\section{Corresponding Author:- Mohamed Rachid Bensalah.}




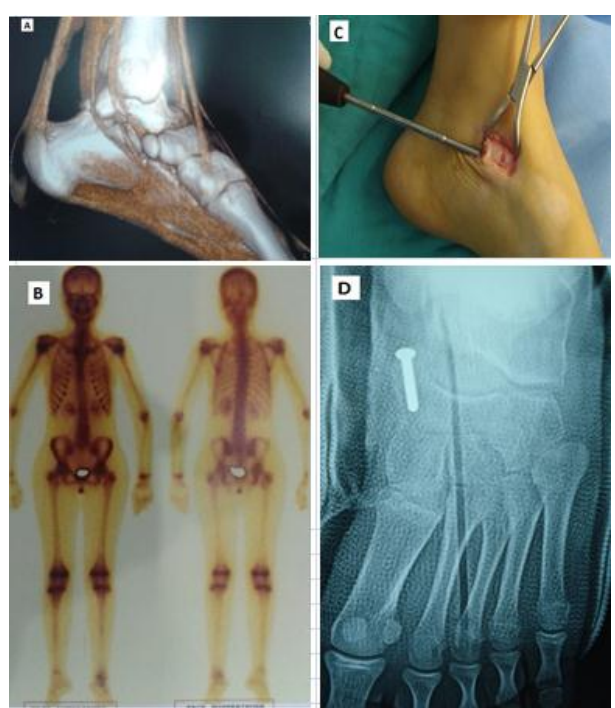

Figure A: Image scanographique montrant un os naviculaire accesoire avec insertion de tendon tibial posterieur. Figure B: Image scintigraphique montrant une hyperfixation de l'os naviculaire.

Figure C: Arthrodése de l'os naviculaire avec son accessoire en peropératoire.

Figure D: Radio de contrôle post opératoire. 